\title{
Assessment of Construct Validity of a Virtual Reality Laparoscopy Simulator
}

\author{
RACHEL ROSENTHAL, MD, ${ }^{1}$ WALTER A. GANTERT, MD ${ }^{1}$ CHRISTIAN HAMEL, MD, ${ }^{1}$ \\ DIETER HAHNLOSER, MD, ${ }^{2}$ JUERG METZGER, MD, ${ }^{3}$ THOMAS KOCHER, MD, FACS, ${ }^{4}$ \\ PETER VOGELBACH, MD, ${ }^{5}$ DANIEL SCHEIDEGGER, MD, ${ }^{1}$ \\ DANIEL OERTLI, MD, FACS, ${ }^{1}$ and PIERRE-ALAIN CLAVIEN, MD, FACS ${ }^{2}$
}

\begin{abstract}
Background: The aim of this study was to assess whether virtual reality (VR) can discriminate between the skills of novices and intermediate-level laparoscopic surgical trainees (construct validity), and whether the simulator assessment correlates with an expert's evaluation of performance.

Methods: Three hundred and seven (307) participants of the 19th-22nd Davos International Gastrointestinal Surgery Workshops performed the clip-and-cut task on the Xitact LS 500 VR simulator (Xitact S.A., Morges, Switzerland). According to their previous experience in laparoscopic surgery, participants were assigned to the basic course (BC) or the intermediate course (IC). Objective performance parameters recorded by the simulator were compared to the standardized assessment by the course instructors during laparoscopic pelvitrainer and conventional surgery exercises.

Results: IC participants performed significantly better on the VR simulator than BC participants for the task completion time as well as the economy of movement of the right instrument, not the left instrument. Participants with maximum scores in the pelvitrainer cholecystectomy task performed the VR trial significantly faster, compared to those who scored less. In the conventional surgery task, a significant difference between those who scored the maximum and those who scored less was found not only for task completion time, but also for economy of movement of the right instrument.

Conclusions: VR simulation provides a valid assessment of psychomotor skills and some basic aspects of spatial skills in laparoscopic surgery. Furthermore, VR allows discrimination between trainees with different levels of experience in laparoscopic surgery establishing construct validity for the Xitact LS 500 clip-and-cut task. Virtual reality may become the gold standard to assess and monitor surgical skills in laparoscopic surgery.
\end{abstract}

\footnotetext{
${ }^{1}$ Departments of Anesthesiology and Surgery, University Hospital, Basel, Switzerland.

${ }^{2}$ Department of Visceral and Transplantation Surgery, University Hospital, Zürich, Switzerland.

${ }^{3}$ Department of Surgery, State Hospital, Luzern, Switzerland.

${ }^{4}$ Department of Surgery, State Hospital, Baden, Switzerland.

${ }^{5}$ Department of Surgery, District Hospital, Dornach, Switzerland.

This work was supported by the Swiss National Science Foundation (SNF \#3200-068307.02) and was presented at the 13th Annual Meeting of the European Surgical Association, Zürich, Switzerland, on April 8th, 2006.
} 


\section{INTRODUCTION}

$\mathbf{T}$ HE INTRODUCTION OF MINIMALLY INVASIVE surgery has dramatically revolutionized surgical treatment. Degraded visual and haptic information, as well as the altered mechanical conditions in laparoscopic surgery, are particularly challenging to the surgeon and require different skills than conventional surgery. Traditional surgical skills training, based on the Halstedian apprenticeship model, is inappropriate for the training of laparoscopic surgical skills. ${ }^{1}$ Various alternative training methods have been developed, employing synthetic models in box trainers, cadaveric animal models, or anesthetized pigs. Box trainers have the disadvantage of insufficient realism and the lack of objective assessment and feedback. The use of animal models is sharply criticized by animal-rights organizations. Economic, ethical, medicolegal and educational considerations have, therefore, expedited the development of computer-based training methods. ${ }^{2}$

Virtual reality (VR) simulation appears to be an ideal tool for the training of laparoscopic surgical skills. The interface between trainee and surgical site, consisting of a video screen and instruments subjected to the socalled fulcrum effect with kinesthetic feedback, can readily be simulated by modern VR simulation technology. The fact that the tip of the instrument moves in the direction opposite to the surgeon's hand, creating a dissonance between visual and proprioceptive feedback, is known as the fulcrum effect of the body wall on instrument manipulation. ${ }^{3}$ One major advantage of the VR simulator lies in its capability of precise and objective measurements of performance parameters. ${ }^{4}$ This permits the investigation of the component skills of laparoscopic surgical performance and their objective assessment. It appears that adequate performance in laparoscopic surgery depends on basic perceptual motor skills (e.g., visual and haptic information acquisition and motor output), cognitive skills (e.g., spatial skills, attention, and knowledge), and noncognitive factors (e.g., physical condition, emotional state, or personality attributes)..$^{5-8}$

A number of studies have, thus far, investigated issues of reliability and validity of skills assessments by using various tasks on a VR simulator. ${ }^{9-12}$ These tasks mainly addressed perceptual motor skills and some basic aspects of spatial skills.

However, the drawback of many studies is the fact that they were done with only a few participants, and most of them were trained at the same institution. Therefore, general conclusions on VR skills assessment must be taken with caution.

The aim of this study was to investigate the construct validity of skills assessment by using a simple, but realistic, task representing one step of laparoscopic chole- cystectomy in the setting of a large international surgical skills training course. We hypothesized that specific performance measurements of the task on the VR simulator could discriminate between surgical trainees with basic skills and trainees with intermediate-level experience, and that the scores would correlate with an expert's structured assessment of the trainee's performance in the pelvitrainer as well as during conventional surgery exercises.

\section{MATERIALS AND METHODS}

\section{Study participants}

Three hundred and seven (307) surgical trainees at four consecutive international surgical skills courses, the 19th-22nd Davos International Gastrointestinal Surgery Workshops (2002-2005), performed a standardized task on the Xitact LS 500 virtual reality simulator (Xitact S.A., Morges, Switzerland), in addition to skills training and assessment in standard pelvitrainer and conventional surgery exercises. The workshop consisted of two parallel courses with mostly separate lectures and practical exercises, a basic course (BC), and an intermediate course (IC). The conventional surgery exercises were equivalent in both courses; the IC participants were given the opportunity to perform some more advanced pelvitrainer exercises. According to their previous experience in laparoscopic surgery, which they had to disclose in a questionnaire prior to the course investigating the number of previously performed laparoscopic interventions, they were assigned to either the BC or the IC. Each study participant signed an informed consent form before entering the VR simulator study.

\section{Simulator task}

Each study participant performed the clip-and-cut task on the Xitact LS 500 virtual patient laparoscopy simulator (Fig. 1). The simulation runs on a $1.0-\mathrm{GHz}$ Pentium III PC with Windows 2000 operating system and a 19inch-high resolution TFT monitor. The computer is connected to two robotic force-feedback devices, which act as bidirectional interfaces, and a third unidirectional electromechanical interface to direct the laparoscope. Real-time graphic and haptic (force-feedback) representations of the operative field, including tissue deformation and collision detection of soft tissues, are provided.

The tasks were performed on any day during the workshop. The task consisted of clipping and cutting the cystic artery and duct (Fig. 2). The task was performed one to three times. The LS 500 clip-and-cut task is a reasonably simple task that can be performed by novices without any prior experience in laparoscopic surgery. Performance relies on the trainee's perceptual motor and some 


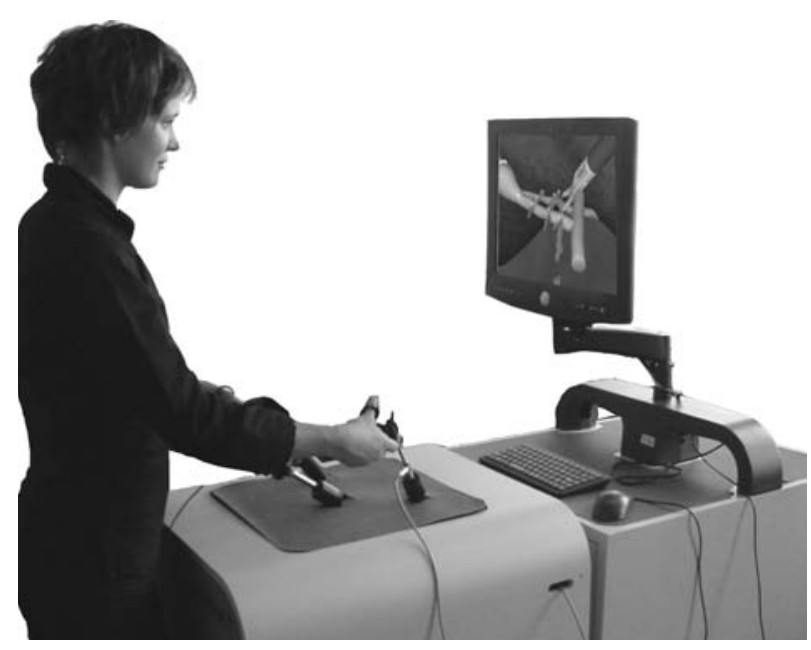

FIG. 1. The LS 500 simulator. The LS 500 Xitact virtual patient laparoscopy simulator was used for the presented test series.

basic aspects of spatial skills, whereas higher cognitive skills (e.g., problem-solving capability) are not necessary. Repetitions were chosen to improve the reliability of the test score.

For each participant and trial, the computer recorded total task completion time and tool tip travel distance (i.e., economy of movement) of the right-hand instrument (clipping/cutting) and the left-hand instrument (retracting the gallbladder) among other performance measurements.

\section{Course evaluation}

The instructions for the course exercises were given by videotapes and live video demonstrations. All instructors were board-certified surgeons of various European countries They evaluated the participants' performance according to a structured observation form $(0=$ persistently unsatisfactory performance; $1=$ frequent errors or occasional errors uncorrected by participant; $2=$ occasional errors, but corrected by participant; and $3=$ no errors observed) during a laparoscopic cholecystectomy on a porcine gallbladder/liver model in a pelvitrainer. Two (2) groups of participants were identified: those with a maximum score in the instructor's pelvitrainer assessment and those who achieved less than the maximum score.

In addition, all course participants concluded the Davos International Gastrointestinal Surgery Workshop with a final practical exam, which consisted of performing an end-to-end small-bowel anastomosis by using a single layer of continuous, extramucosal, absorbable suture on the porcine small bowel in a conventional surgical setting. Performance during this practical exam was assessed according to a 16-point task checklist (Table 1) and rated with: $0=$ task not done, incorrect; and $1=$ task done, correct for every task. Again, the group of participants with the maximum score was distinguished from the group with the less than maximum score for further investigation.

Objective performance parameters recorded by the simulator were correlated with the category of performance assessment during the pelvitrainer laparoscopic cholecystectomy and during the final exam.

\section{Statistical analysis}

All data were entered into a Microsoft Excel 2003 (Microsoft Corporation, Redmond, WA) spreadsheet, and SPSS software version 10.1 (SPSS Inc., Chicago, IL) was used for statistical investigation. Owing to nonnormal distribution of the data, the Mann-Whitney U Test was applied for a statistical analysis of performance. The difference in experience in surgery between BC and IC participants was evaluated by using a chi-square test. A $P$-value of less than 0.05 was considered statistically significant.

\section{RESULTS}

A total of 307 surgical trainees from 17 different countries participated in the trial, and 157 participants were assigned to the $\mathrm{BC}$ and 150 to the IC group.

Among the participants assigned to the $\mathrm{BC}$ group, $86.3 \%$ were first- or second-year residents and $13.7 \%$ were third- to fifth-year residents. The IC trainees participated at the course in $32.6 \%$ after over 5 years of clinical experience, in $65.3 \%$ they were third- to fifth-year residents, and in $2.1 \%$ first- or second-year residents. This difference was significant $(P<0.001)$. Moreover, the IC

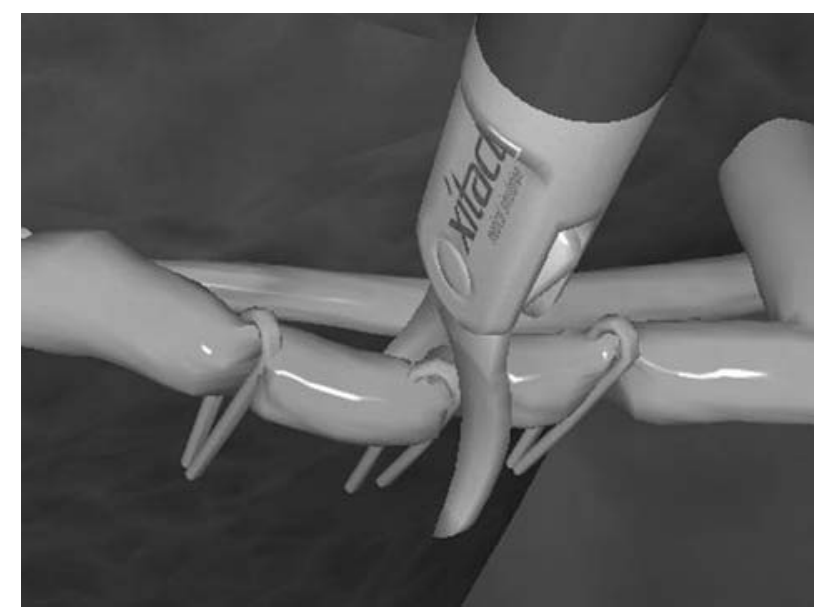

FIG. 2. The clip and cut task. The standardized task consisted in clipping and cutting the cystic artery and the cystic duct. 
Table 1. Task Checklist for Evaluation of the Final Practical Exam Consisting of an End-To-End Small Bowel Anastomosis

Bowel oriented mesenteric border to mesenteric border Uses stay sutures to stabilize the bowel

Needle loaded $1 / 2$ to $2 / 3$ from tip

Needle enters bowel at right angles most of the time

Single attempt at needle passage through bowel most of the time

Follow through on curve of needle most of the time

Forceps used on seromuscular layer of bowel only

Uses instruments to handle needle most of the time

Satisfactory inverting extramucosal suture technique

Even sutures spaced 3-5 mm apart

Square knots with at least three throws each (instrument- or hand tied)

Sutures cut to appropriate length (approximately $0.5 \mathrm{~cm}$ )

No mucosal pouting

No anastomotic narrowing or purse-string effect

Good apposition of bowel without excessive tension on sutures

Good use of assistant throughout exercise

participants had previously performed significantly more laparoscopic cholecystectomies $(P<0.001)$, appendectomies $(P<0.001)$, and fundoplications $(P=0.010)$ (Table 2).

Eighty (80) participants were women (26\%) and 227 were men (74\%). All participants performed the simulator task once (157 BC, 150 IC), 219 twice (122 BC, 97 IC), and 74 three times (39 BC, 35 IC). Two hundred and ninety-five (295) of the 307 trainees who participated in our simulator study completed the pelvitrainer laparoscopic cholecystectomy task, and 296 of 307 accomplished the final exam.

There was no significant difference in simulator task performance between male and female participants.

In average, IC participants performed better than BC participants in the VR simulator tasks. This difference was significant for the task completion time as well as for economy of movement of the right-hand instrument, but not for the left-hand instrument (Table 3).

The performance parameters of all participants who did repetitive trials improved in the second trial as well as in the third trial, when compared to the first trial. However, performance improved from the second to the third trial only in the IC group, not in the BC group.

In a subgroup analysis of those participants who performed three trials, these findings were confirmed. In this subgroup, we found that IC participants performed significantly better than $\mathrm{BC}$ participants (task completion time for trial $1 \mathrm{BC}$ : 251 seconds versus IC: 184 seconds, $P=0.003$; for trial $2 \mathrm{BC}: 204$ seconds versus IC: 161 seconds, $P=0.043$; for trial $3 \mathrm{BC}$ : 216 seconds versus IC: 155 seconds, $P=0.003$; economy of movement right instrument: for trial $1 \mathrm{BC}: 2.6 \mathrm{~m}$ versus $\mathrm{IC}: 1.7 \mathrm{~m}, P=$ 0.002; for trial $2 \mathrm{BC}: 2.2 \mathrm{~m}$ versus IC: $1.7 \mathrm{~m}, P=0.045$; for trial $3 \mathrm{BC}: 2.2 \mathrm{~m}$ versus IC: $1.6 \mathrm{~m}, P=0.002$; economy of movement left instrument: for trial $1 \mathrm{BC}: 1.6 \mathrm{~m}$ versus IC: $1.2 \mathrm{~m}, P=0.100$; for trial $2 \mathrm{BC}: 1.3 \mathrm{~m}$ versus IC: $0.9 \mathrm{~m}, P=0.065$; for trial $3 \mathrm{BC}: 1.4 \mathrm{~m}$ versus IC: $1.0 \mathrm{~m}, P=0.131)$.

Participants with a maximum score $(3 / 3$ points) in the pelvitrainer laparoscopic cholecystectomy task $(n=149)$ performed the first two VR simulator task trials significantly faster when compared to the group of participants who scored less than maximum $(n=146)$ (Table 4$)$. However, there was no correlation of economy of movement of either instrument in the simulator task with the instructor's assessment of pelvitrainer performance. The third simulator trial was excluded from analysis owing to the small numbers of participants in the subgroups. A significant difference was found in task completion time in the first and second trial of the simulator task between those who scored maximum $(16 / 16$ points; $n=182)$ and those who scored less than maximum $(n=114)$ in the final exam. In contrast to the pelvitrainer assessment, a

Table 2. Number of Previously Performed Laparoscopic Interventions for Basic and Intermediate Course Participants

\begin{tabular}{|c|c|c|c|c|c|c|}
\hline \multirow[b]{3}{*}{ No } & \multicolumn{2}{|c|}{ Cholecystectomy } & \multicolumn{2}{|c|}{ Appendectomy } & \multicolumn{2}{|c|}{ Fundoplication } \\
\hline & $B C$ & $I C$ & $B C$ & $I C$ & $B C$ & $I C$ \\
\hline & $\%$ & $\%$ & $\%$ & $\%$ & $\%$ & $\%$ \\
\hline 0 & 80.4 & 04.2 & 84.3 & 16.2 & 100 & 92.9 \\
\hline $1-5$ & 13.7 & 16.9 & 11.1 & 11.8 & - & 5.5 \\
\hline $6-10$ & 03.9 & 09.9 & 03.3 & 18.4 & - & 0.8 \\
\hline $11-20$ & 02.0 & 35.2 & 01.3 & 27.9 & - & 0.8 \\
\hline \multirow[t]{2}{*}{$>20$} & - & 33.8 & - & 25.7 & - & - \\
\hline & \multicolumn{2}{|c|}{$<0.001^{\mathrm{a}}$} & \multicolumn{2}{|c|}{$<0.001^{\mathrm{a}}$} & \multicolumn{2}{|c|}{$<0.001^{\mathrm{a}}$} \\
\hline
\end{tabular}

$\mathrm{BC}$, basic course; IC, intermediate course; No, number of interventions performed prior to course, data given in percent of participants who had previously performed the indicated number of interventions.

${ }^{\mathrm{a}} P$-value for the difference between BC and IC $(P<0.05)$. 
Table 3. Performance of the BC and IC Participants at Trial 1, 2, and 3 (Mean \pm Standard Deviation)

\begin{tabular}{|c|c|c|c|c|c|c|}
\hline \multirow[b]{2}{*}{ No } & \multicolumn{2}{|c|}{ Time $(s)$} & \multicolumn{2}{|c|}{$L$ instrument $(m)$} & \multicolumn{2}{|c|}{$R$ instrument $(m)$} \\
\hline & $B C$ & $I C$ & $B C$ & IC & $B C$ & IC \\
\hline 1 & $223( \pm 101)$ & $177( \pm 83)$ & $1.5( \pm 1.6)$ & $1.2( \pm 1.0)$ & $2.4( \pm 1.2)$ & $1.9( \pm 0.9)$ \\
\hline 2 & $179( \pm 80)$ & $161( \pm 77)$ & $1.2( \pm 0.9)$ & $1.1( \pm 1.1)$ & $2.0( \pm 1.0)$ & $1.7( \pm 0.8)$ \\
\hline 3 & $216( \pm 99)$ & $\begin{array}{l}155( \pm 61) \\
03^{\mathrm{a}}\end{array}$ & $1.4( \pm 1.6)$ & $1.0( \pm 1.1)$ & $2.2( \pm 1.0)$ & $\frac{1.6( \pm 0.5)}{92^{\mathrm{a}}}$ \\
\hline
\end{tabular}

${ }^{\text {a }} P$-value for the difference between $\mathrm{BC}$ and IC $(P<0.05)$.

No, trial number; Time, total task completion time in seconds; L/R instrument, left/right instrument travel distance in meters; $\mathrm{BC}$, basic course; IC, intermediate course.

significant difference was also found for economy of movement of the right-hand instrument (Table 5). No difference was found for tool tip travel distance of the lefthand instrument. The third trial was again excluded from analysis owing to insufficient statistical power.

\section{DISCUSSION}

To our knowledge, with 307 international participants, this was the largest surgical simulator validation study to demonstrate that specific performance measurements in a standardized task on a virtual reality simulator can objectively discriminate between novices and intermediatelevel trainees in laparoscopic surgery and establishing construct validity for the Xitact LS 500 clip-and-cut task, and that the simulator performance assessment correlates with an expert's structured evaluation of performance in a pelvitrainer laparoscopic task as well as during an exercise of conventional surgery.

The findings from this study prove construct validity of assessment of perceptual motor and some of the basic aspects of spatial skills using the Xitact LS 500 clip-andcut task. The capability of the simulator assessment to discriminate between 37 expert surgeons $(>100$ laparoscopic cholecystectomies) and 37 novices (no laparoscopic surgery experience) on the same Xitact LS 500 task has previously been shown. ${ }^{11}$ The experts were significantly faster than the novices and reached higher overall scores. In contrast, however, we did not compare novices to experts, but two groups of trainees with more or less previous clinical experience, and found a significant difference in task completion time and economy of movement of the right-hand instrument. We have, therefore, shown that simulator performance measurements can discriminate not only between expert and novice surgeons, but also between surgical trainees with different levels of experience. Moreover, with 307 testees, this was demonstrated with a very high number of study participants, which was a novel step in surgical simulator validation.

The fact that only economy of movement of the righthand instrument showed a significant difference between the groups, and not of the left-hand instrument, may be explained by the fact that the left-hand instrument has a more static role than the right-hand instrument, rather than the fact that $92 \%$ of the participants were right-handed. Therefore, measurement of the left-hand instrument path may be affected by a floor effect and would not be able to detect a difference in performance. Conversely, it may also point out that an unexperienced laparoscopic surgeon uses his or her left-hand instrument not more or even less for exposure than a more experienced surgeon.

Table 4. Correlation of the Performance at the Pelvitrainer-Based Sessions and of the Performance at the Simulator Trials 1 and 2 (Mean \pm Standard Deviation)

\begin{tabular}{|c|c|c|c|c|c|c|}
\hline \multirow[b]{2}{*}{ No } & \multicolumn{2}{|c|}{ Time $(s)($ mean $\pm S D)$} & \multicolumn{2}{|c|}{$L$ instrument $($ mean $\pm S D)$} & \multicolumn{2}{|c|}{$R$ instrument $($ mean $\pm S D)$} \\
\hline & $C_{\max }$ & $C_{\text {less }}$ & $C_{\max }$ & $C_{\text {less }}$ & $C_{\max }$ & $C_{\text {less }}$ \\
\hline \multirow[t]{2}{*}{1} & $188( \pm 194)$ & $211( \pm 94)$ & $1.3( \pm 1.4)$ & $1.3( \pm 1.1)$ & $2.1( \pm 1.2)$ & $2.2( \pm 1.0)$ \\
\hline & \multicolumn{2}{|c|}{$0.034^{\mathrm{a}}$} & \multicolumn{2}{|c|}{0.183} & \multicolumn{2}{|c|}{0.223} \\
\hline 2 & $0.005^{\mathrm{a}}$ & $183( \pm 78)$ & 0.065 & $1.2( \pm 0.9)$ & 0.157 & $1.9( \pm 0.9)$ \\
\hline
\end{tabular}

${ }^{\text {a }} P$-value for the difference between $\mathrm{C}_{\max }$ and $\mathrm{C}_{\text {less }}(P<0.05)$.

No, trial number; Time, total task completion time in seconds; L/R instrument, left/right instrument travel distance in meters; $\mathrm{C}_{\max }$, participants with maximum scores in the pelvitrainer cholecystectomy task $(n=149)$; $\mathrm{C}_{\text {less }}$, participants with less than maximum score $(n=146)$. 
Table 5. Correlation of the Final Assessment and of the Performance at the Simulator Trials 1 and 2 (Mean \pm Standard Deviation)

\begin{tabular}{|c|c|c|c|c|c|c|}
\hline \multirow[b]{2}{*}{ No } & \multicolumn{2}{|c|}{ Time $(s)($ mean $\pm S D)$} & \multicolumn{2}{|c|}{$L$ instrument (mean $\pm S D)$} & \multicolumn{2}{|c|}{$R$ instrument (mean $\pm S D)$} \\
\hline & $F_{\max }$ & $F_{\text {less }}$ & $F_{\max }$ & $F_{\text {less }}$ & $F_{\max }$ & $F_{\text {less }}$ \\
\hline 1 & $191( \pm 90)$ & $217( \pm 102)$ & $1.4( \pm 1.4)$ & $1.4( \pm 1.3)$ & $2.0( \pm 1.1)$ & $2.3( \pm 1.1)$ \\
\hline 2 & $158( \pm 70)$ & $193( \pm 90)$ & $1.1( \pm 1.0)$ & $1.2( \pm 1.1)$ & $1.8( \pm 0.9)$ & $2.1( \pm 1.0)$ \\
\hline
\end{tabular}

${ }^{a} P$-value for the difference between $\mathrm{F}_{\max }$ and $\mathrm{F}_{\text {less }}(P<0.05)$.

No, trial number; Time, total task completion time in seconds; L/R instrument, left/right instrument travel distance in meters; $\mathrm{F}_{\max }$, participants with maximum scores in final assessment $(n=182) ; \mathrm{F}_{\text {less }}$, participants with less than maximum score $(n=114)$.

In this study, the performance parameters of all participants who did repetitive trials improved in the second trial as well as in the third trial when compared to the first trial. This is in accordance with kinesiologic theory, which states that improvement of motor performance over repetitive trials on a specific task follows a logarithmic function. ${ }^{13}$

However, performance improved from the second to the third trial only in the IC group, not in the BC group. Schijven et al. have found similar results. In their study, the increase in score over runs of VR trials was significant only in the expert group, but not in the novices group. ${ }^{11}$

It was certainly a drawback of our study that not all of the 307 participants performed three trials of the simulator task. This may have led to a selection bias. However, this was caused by time constraints and some organizational difficulties in a large international training course. For the same reason, we did not use a more detailed assessment form and did not have more than one expert evaluate the participants' performance and could not provide any inter-observer variation data.

Our study showed that one simulator performance parameter, task completion time, correlated well with the instructor's evaluation of performance in a pelvitrainer laparoscopic cholecystectomy. A linear correlation of simulator performance with pelvitrainer evaluation was not found, as the pelvitrainer evaluation, although semiquantitative, was characterized by a strong ceiling effect, with almost half of our study participants receiving the maximum score. We, therefore, chose the two groups of maximum or less than maximum score in the pelvitrainer evaluation and found a significant correlation of these two categories with task completion time in the simulator task.

A similar phenomenon was encountered with the final exam, using the conventional surgical task of performing a small-bowel anastomosis. Again, owing to a strong ceiling effect of final exam scores, we formed the two groups with maximum and less than maximum score as criterion categories. We found a good correlation between task completion time in the simulator task and final exam score. In contrast to the pelvitrainer evaluation, we also found a correlation with economy of movement of the right-hand instrument (significant for two parameters instead of only one). This may have been owing to the more detailed structured evaluation in the final exam, which led to a more precise criterion measurement.

The correlation of simulator task performance measurement with scores on the final exam is remarkable, as it points out that perceptual motor skills and some aspects of spatial skills may be equally important for a laparoscopic, as well as for a conventional, surgical task. It may well be that the particular perceptual motor skills (i.e., eye-hand coordination) for adequate performance in laparosopic surgery have, thus far, been overestimated, and that there are greater differences in some complex spatial skills and higher cognitive skills between laparoscopic and conventional open surgery.

Beyond skills assessment, several research groups have demonstrated the value of VR simulation for improving a trainee's laparoscopic surgical skills. In a study comparing a self-directed video trainer and VR training systems on acquisition of laparoscopic skills, it was shown that psychomotor skills improve after training on both systems, whereas operative performance during laparascopic cholecystectomy improved only in the VR training group. ${ }^{14}$ Moreover, two randomized trials have confirmed that VR training improves performance in the operating room. ${ }^{15,16}$ Today, we are only beginning to identify all the necessary component skills for adequate performance in laparoscopic surgery. It may well be that the VR simulator holds yet its greatest potential in the identification of all component skills, which will lead to further refinement of laparoscopic surgical skills training curricula in the future.

The eventual goal of surgical simulation will be to substitute for valuable training time in the operating room. The calculation of so-called isoperformance curve, as 
known from aviation training (as hours on the simulator are increased, hours of in-flight training may be reduced), will give us ultimate proof of the simulator's impact on surgical skills training. ${ }^{17,18}$

\section{CONCLUSIONS}

In the largest surgical simulator validation study to date, we have demonstrated that VR simulator performance assessments are a valid assessment of perceptual motor skills and basic spatial skills in laparoscopic surgery. They allow for the discrimination between novices and laparoscopic surgical trainees with intermediate experience. Trainees can improve their skills quickly. VR may become the gold standard to assess and monitor surgical skills in laparoscopic surgery.

\section{REFERENCES}

1. Halsted WS. The training of the surgeon. Bull Johns Hop Hosp 1904;15:267-275.

2. Aggarwal R, Moorthy K, Darzi A. Laparoscopic skills training and assessment. Br J Surg 2004;91:1549-1558.

3. Gallagher AG, McClure N, McGuigan J, Ritchie K, Sheehy NP. An ergonomic analysis of the fulcrum effect in the acquisition of endoscopic skills. Endoscopy 1998;30:617620.

4. Haluck RS, Krummel TM. Computers and virtual reality for surgical education in the 21st century. Arch Surg 2000; 135:786-792.

5. Adrales GL, Donnelly MB, Chu UB, Witzke DB, Hoskins JD, Mastrangelo, Jr, MJ, Gandsas A, Park AE. Determinants of competency judgments by experienced laparoscopic surgeons. Surg Endosc 2004;18:323-327.

6. Graham KS, Deary IJ. A role for aptitude testing in surgery? JR Coll Surg Edinb 1991;36:70-74.

7. Hanna GB, Drew T, Clinch P, Hunter B, Shimi S, Dunkley MP, Cuschieri A. A microprocessor-controlled psychomotor tester for minimal access surgery. Surg Endosc 1996;10:965-969.

8. Vincent C, Moorthy K, Sarker SK, Chang A, Darzi AW. Systems approaches to surgical quality and safety: From concept to measurement. Ann Surg 2004;239:475-482.
9. Gallagher AG, Lederman AB, McGlade K, Satava RM, Smith CD. Discriminative validity of the minimally invasive surgical trainer in virtual reality (MIST-VR) using criteria levels based on expert performance. Surg Endosc 2004;18:660-665.

10. Schijven M, Jakimowicz J. The learning curve on the Xitact LS 500 laparoscopy simulator: Profiles of performance. Surg Endosc 2004;18:121-127.

11. Schijven M, Jakimowicz J. Construct validity: Experts and novices performing on the Xitact LS500 laparoscopy simulator. Surg Endosc 2003;17:803-810.

12. Gallagher AG, Richie K, McClure N, McGuigan J. Objective psychomotor skills assessment of experienced, junior, and novice laparoscopists with virtual reality. World J Surg 2001;25:1478-1483.

13. Schmidt, RA. Motor Control and Learning: A Behavioral Emphasis. Champaign, IL: Human Kinetics Publishers, 1982.

14. Hamilton EC, Scott DJ, Fleming JB, Rege RV, Laycock R, Bergen PC, Tesfay ST, Jones DB. Comparison of video trainer and virtual reality training systems on acquisition of laparoscopic skills. Surg Endosc 2002;16:406-411.

15. Seymour NE, Gallagher AG, Roman SA, O'Brien MK, Bansal VK, Andersen DK, Satava RM. Virtual reality training improves operating room performance. Ann Surg 2002;236:458-464.

16. Grantcharov TP, Kristiansen VB, Bendix J, Bardram L, Rosenberg J, Funch-Jensen P. Randomized, clinical trial of virtual reality simulation for laparoscopic skills training. $\mathrm{Br}$ J Surg 2004;91:146-150.

17. Jones MB, Kennedy RS. Isoperformance curves in applied psychology. Hum Factors 1996;38:167-182.

18. Roscoe SN. Transfer and cost-effectiveness of groundbased flight trainers. In: Roscoe SN (ed). Aviation Psychology. Ames IA: Iowa State University Press, 1980: 194-203.

Address reprint requests to: Rachel Rosenthal, MD

Department of Visceral Surgery Lausanne University Hospital CHUV

Rue du Bugnon 46

1011 Lausanne

Switzerland

E-mail: Rachel.Rosenthal@chuv.ch 